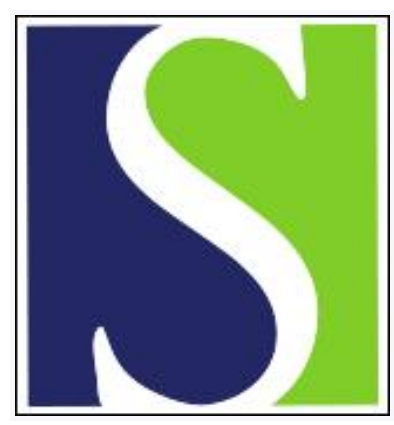

Scand J Work Environ Health 1998;24(6):449-464

https://doi.org/10.5271/sjweh.369

Issue date: Dec 1998

\title{
Work-related low-back problems in nursing
}

by Lagerström M, Hansson T, Hagberg M

The following articles refer to this text: 2001;27(6):388-394;

2002;28(3):168-175; 2002;28(6):386-393; 2003;29(6):468-477

Key terms: ergonomic intervention; nurse; physical factor; psychosocial factor; review; work organizational factor

This article in PubMed: www.ncbi.nlm.nih.gov/pubmed/9988087

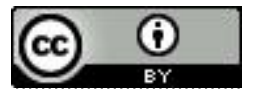




\title{
Work-related low-back problems in nursing
}

\author{
by Monica Lagerström, PhD, ${ }^{1}$ Tommy Hansson, MD, ${ }^{2}$ Mats Hagberg, $M D^{3}$
}

\begin{abstract}
Lagerström M, Hansson T, Hagberg M. Work-related low-back problems in nursing. Scand J Work Environ Health 1998;24(6):449-464
\end{abstract}

This scientific literature review focuses on the relation between nursing work and low-back problems. Its aim was to estimate the risk of physical, psychosocial, and work organizational exposure factors that may lead to low-back problems. In addition this paper reviews and evaluates reported ergonomic intervention, with the object of decreasing the prevalence and incidence of low-back problems among nurses. A considerable number of studies of nursing staff has shown the connection between lifts and transfers of patients on one hand and low-back problems on the other. Factors in nursing work that may be significant in this connection are staff density and work satisfaction. In this review the single individual factor that was indisputably related to low-back problems was "history of back problems". Prevention programs do not show unequivocally positive results. There is a great need to carry out prospective studies with preventive programs.

Key terms ergonomic intervention, nurses, physical factors, psychosocial factors, review, work organizational factors.

\section{Background}

In several respects nursing work is not comparable with other types of work. The most significant factor is patient handling (ie, close contact with another human being in need of help and support), which is considered rewarding but also demanding for the nurse. The work can be physically strenuous, but usually with less repetitive load than, for example, work on an assembly line (1) and with less frequent lifting than work in, for example, warehouses. Furthermore, work postures are often awkward, and nurses walk and stand more than warehouse workers (2). It is not unusual that nurses stand and walk for more than 6 hours a day (3).

Hospitals are high-stress employers $(4,5)$. The organization is hierarchical, and a nurse has to adapt to several supervisory levels, as well as to the demands of the patients. Factors in the work situation that lead to stress on nurses are, for example, violence and threats of violence (6), close contact with suffering and death (7), role ambiguity (8), understaffing (7), and shift work (9). A large proportion of nurses suffers from moderate to severe "burn-out" (5).

It has been pointed out that the rapid rise in sick leave and early retirement from work due to musculoskeletal disorders during recent decades has not yet been explained convincingly (10). High levels of work-related low-back problems are found among nursing personnel, and, since nursing is primarily a female career and a large proportion of the female work force consists of nursing personnel, this is a prominent problem in worklife. The 1 -year prevalence of low-back problems among 1616 English nurses (mean age 38 years) was $45 \%$ (11), which can be compared with the 1-year prevalence rate of $35 \%$

National Institute for Working Life, Solna, Sweden.

Department of Orthopaedics, Sahlgren University Hospital, Gothenburg University, Göteborg, Sweden.

Department of Occupational Medicine, Sahlgren University Hospital, Gothenburg University, Göteborg, Sweden.

Reprint requests to: Dr M Lagerström, National Institute for Working Life, S-171 84 Solna, Sweden. [E-mail: monica.lagerstrom@niwl.se] 
for Danish women (mean age 41 years) in the general population (12). Among the English nurses the life-time prevalence was $60 \%$, and this figure could be compared with $45-64 \%$ for 1495 women from 8 areas in Great Britain in all age ranges (20-59 years) (11).

When different vocational groups among nurses are defined, there are often differences in the prevalence of low-back problems among the groups. Generally speaking, fewer registered nurses than state enrolled nurses, or auxiliary nurses (nurses' aides) report low-back problems. In a Swedish study of 688 nursing personnel the point prevalence of ongoing low-back problems was $52 \%$ for registered nurses, of whom $14 \%$ experienced severe problems. For state enrolled nurses the corresponding numbers were $55 \%$ and $15 \%$, and for auxiliary nurses, they were $65 \%$ and $22 \%$, respectively (13). The issue of low-back problems and their relation to work often arises. In a study of 348 female nurses, about 4 out of $5(80 \%)$ considered these problems to be related to work (14). Thus the prevalence of low-back problems seems to be slightly higher for nurses than for women of a similar age range in the general population. However, the magnitude of this difference, especially among registered nurses, is smaller than is commonly perceived. A register study showed, however, that the risk for occupational accidents of the back was higher among nurses than among working women in the general population (15).

Because of the rise in sick leave due to low-back problems among nurses, several studies have focused on identifying work-related risk factors to point to the route of predicator intervention. Hence ergonomic intervention has been described and evaluated in some studies. Intervention generally focuses on the individual person and often consists of training programs in lifting and handling techniques; it has proved to be of little long-term benefit (16). There is agreement as regards the need for intervention that leads to a reduction in the prevalence and incidence of low-back problems among nurses.

\section{Aim of the study}

The aim of the review was to assess the reported knowledge on the relationship between nursing work and lowback problems, and, further, to make a risk assessment of the influence of physical, psychosocial, and work organizational factors that may lead to low-back problems.

On the basis of previous epidemiologic studies and the scientific literature on work at hospitals, the following factors were considered possible work-related risk factors for low-back problems in nursing work: (i) physical factors: lifts and transfers and "save the patient" situations, work postures, work category, and clinical area; (ii) psychosocial factors: psychological demands, authority over decisions, skill utilization, social support at work, work satisfaction, and stress; and (iii) work organization- al factors: staff density, shift work, mode of nursing care, and work technique.

A further aim of this paper was to review and evaluate reported ergonomic intervention with the objective of decreasing the prevalence and incidence of low-back problems among nurses. A main object was to find relevant ergonomic intervention in nursing work that might result in the prevention of low-back problems.

\section{Selection of the literature}

An extensive search was made of the scientific literature published from 1988 to 1998 for studies on low-back problems among health personnel. The focus on the last 10 years was chosen because of the rapidly changing work situation at hospitals, nursing homes, and other types of nursing institutions. The key words "nurses", "nurses" aides", and "back pain" were used on computer-based bibliographic databases such as NIOSHTIC, Medline, Arbline, and Spiline. Searches were also carried out in reference lists of review articles and other scientific "peer-reviewed" articles, via contacts with researchers within the field, as well as in the personal archives and computer-based data bases of the authors. When there were several reports based on the same study, the one or two most relevant to the purposes of this review were retained.

Included in the study were peer-review articles as follows: (i) studies of the relationship between low-back problems and exposure and (ii) intervention studies.

Most of the studies were of cross-sectional design $(\mathrm{N}=21)$ (table 1), 10 studies presented prospective data (table 2), and 11 concerned intervention programs (table 3 ). In the longitudinal studies and in the intervention studies at least 2 data collections had to be reported.

\section{Quality comments}

All the studies that were obtained were further reviewed for methodological quality issues and descriptive information as follows (for each study see tables 1, 2, and 3):

1. Among nursing personnel there are different occupational groups of "nurses": untrained nursing aides, state enrolled nurses, and registered nurses. Due to different tasks and exposure, the groups ought to be defined to clear the generalizability of the results.

2 . Before the work situation of nurses can be evaluated, not only physical exposures, but also psychosocial exposures need to be clearly assessed. Bongers et al (17) suggested that psychosocial factors could directly influence mechanical load through changes in postures and the like, since the 2 types of exposure are not independent on each other.

3. Low-back problems must be defined. This item could be either primary data of (low) back problems reported in validated questionnaires [eg, the Nordic 
Table 1. Work-related risk factors for low-back problems in nursing - cross-sectional and case-referent studies. (OR $=$ odds ratio, $R R=$ rate ratio, $95 \% \mathrm{Cl}=95 \%$ confidence interval, OWAS = Ovako Working Posture Analysing System, NS = not significant; incomplete information: $\mathrm{S}=$ sample, $\mathrm{PhE}=$ physical exposure, $\mathrm{PSE}=$ psychosocial exposure, $\mathrm{LBP}=$ low-back problems, $\mathrm{PR}=$ participation rate $<70 \%, \mathrm{IR}=$ incidence density ratio)

\begin{tabular}{|c|c|c|c|c|c|}
\hline Reference & Population & $\begin{array}{l}\text { Data } \\
\text { collection }\end{array}$ & $\begin{array}{l}\text { Quality } \\
\text { comments }\end{array}$ & $\begin{array}{l}\text { Work-related } \\
\text { risk factors }\end{array}$ & $\begin{array}{l}\text { Outcome } \\
\text { measure }\end{array}$ \\
\hline $\begin{array}{l}\text { Jensen, } \\
1990(20)\end{array}$ & $\begin{array}{l}\text { Health care personnel } \\
\text { from the United States, } \\
\text { Scotland, Canada and } \\
\text { Australia }\end{array}$ & $\begin{array}{l}\text { Meta-analyses; } \\
1 \text { prospective } \\
\text { cohort and } 5 \text { cross- } \\
\text { sectional studies }\end{array}$ & $\begin{array}{l}\text { PhE, PSE, } \\
\text { LBP, PR }\end{array}$ & $\begin{array}{l}\text { a) Frequent patient } \\
\text { care, b) infrequent } \\
\text { patient care }\end{array}$ & $\begin{array}{l}\text { LBP accordi } \\
\text { the definitio } \\
\text { the different }\end{array}$ \\
\hline $\begin{array}{l}\text { Jörgensen } \\
\text { et al, } 1994 \\
\text { (21) }\end{array}$ & $\begin{array}{l}28619 \text { assistant nurses } \\
\text { (40\% working in hospi- } \\
\text { tals and } 60 \% \text { in nursing } \\
\text { homes) compared with } \\
\text { the overall Danish fe- } \\
\text { male populationa }\end{array}$ & $\begin{array}{l}\text { Register data in } \\
1988 ; \text { the assistant } \\
\text { nurses had been } \\
\text { working as such at } \\
\text { least } 4 \text { years prior to } \\
\text { the study }\end{array}$ & PhE, PsE & $\begin{array}{l}\text { Heavy lifting and } \\
\text { strong physical } \\
\text { work loads at work }\end{array}$ & $\begin{array}{l}\text { Operations } f \\
\text { niated lumb } \\
(N=1091 ; r e \\
\text { data })\end{array}$ \\
\hline $\begin{array}{l}\text { Smedley et } \\
\text { al, } 1995 \\
(11)\end{array}$ & $\begin{array}{l}1659 \text { nurses ( } 3 \% \text { men) } \\
\text { employed in the United } \\
\text { Kingdom }\end{array}$ & Questionnaire & PsE, PR & $\begin{array}{l}\text { Frequency of } \\
\text { occupational } \\
\text { activities }\end{array}$ & $\begin{array}{l}\text { LBP lasting } \\
>1 \text { day }\end{array}$ \\
\hline
\end{tabular}

Cato et al, 53 nurses in geriatric,

1989 (24) orthopedic and rehabilitation wards at 1 hos-

pital in the United States
Leighton \& 1134 nursing staff from

(25) ed references among and reference the general population groups

in the United Kingdom

Engels et al, 846 nurses in 4 Dutch Questionnaire

1996 (28) nursing homes
Questionnaire

S, PSE
PsE

S, PSE, PR Use of lifting devices during patien transfers

Availability and use Occupational $L B P$ of mechanical lifting defined as LBP aids; identification related to work on of unsafe equipment present unit

Work pressure, pos- Back problems

\section{Main findings}

a) prevalence rate $=3.7$, b) pre-

valence rate $=1$

Risk of operation of herniated lum bar disc among assistant nurses / overall female population, $O R=1.6$ $(95 \%$ Cl $1.2-2.2, P<0.01)$

Manually move a patient on the bed 5-9 times per shift, $\mathrm{OR}=1.5(95 \%$ Cl 1.1-2.2); manually transfer a patient between bed and chair 5-9 times per shift, $0 R=1.7(95 \% \mathrm{C}$

1.2-2.3); transfer a patient between bed and chair with a hoist $>5$ times per shift, $O R=N S$; manually

lift a patient up off the floor $>1$ time per shift, $O R=1.3(95 \% \mathrm{Cl} 1.0-1.6)$ (adjusted for age and height)

Differences between nurses with or without $L B P$, years in present unit $(6.3 / 2.5, P<0.05)$; lifting a patient in bed and one-person transfer were activities mostly associated with LBP

Incidence and prev- $48 \%$ of 222 nurses with back alence of back prob-problems implicated that positionlems and sick leave ing a patient in bed initiated back problems tures, lifting, ergonomic layout of (arm, neck and leg ward problems)

Physical stress: $0 \mathrm{R}=1.99(95 \% \mathrm{Cl}$ $1.30-3.04$ ) for awkward posture, $\mathrm{OR}=2.22(95 \% \mathrm{Cl} 4.46-3.40)$ for stooping, $\mathrm{OR}=2.20(95 \% \mathrm{Cl} 1.38$ $3.50)$ for lifting heavy burdens; work pressure: OR=1.94 $(95 \% \mathrm{Cl} 1.20-$

3.14) for ought to slow down (adjusted for age, gender, duration of employment, workhours per week, and having managerial tasks)

\begin{tabular}{|c|c|c|c|}
\hline $\begin{array}{l}\text { Lee \& } \\
\text { Chiou, } \\
1994(30)\end{array}$ & $\begin{array}{l}3159 \text { female nursing } \\
\text { personnel in } 4 \text { hos- } \\
\text { pitals in Taiwan }\end{array}$ & $\begin{array}{l}\text { Questionnaire, } \quad \text { PSE } \\
\text { experiment with } \\
\text { simulated patient } \\
\text { handling task }(N=24)\end{array}$ & Work postures \\
\hline $\begin{array}{l}\text { Estryn- } \\
\text { Behar et al, } \\
1990(3)\end{array}$ & $\begin{array}{l}1505 \text { female health care } \\
\text { workers at } 26 \text { wards in } \\
\text { Paris }\end{array}$ & $\begin{array}{l}\text { Questionnaire, med-PSE } \\
\text { ical examination, } \\
\text { and records over } \\
\text { number of days of } \\
\text { illness in the } \\
\text { previous year }\end{array}$ & $\begin{array}{l}\text { Work conditions, } \\
\text { posture and lifting } \\
\text { index: a) low index } \\
\text { scores, b) medium } \\
\text { index scores, c) high } \\
\text { index scores }\end{array}$ \\
\hline $\begin{array}{l}\text { Fuortes et } \\
\text { al, } 1994 \\
(31)\end{array}$ & $\begin{array}{l}\text { All nurses at } 1 \text { hospital } \\
\text { during } 2 \text { years; } 100 \\
\text { back injuries and } 197 \\
\text { referents (United States) }\end{array}$ & $\begin{array}{l}\text { Questionnaire } \\
\text { (case-referent) }\end{array}$ & Work practices \\
\hline $\begin{array}{l}\text { Larese \& } \\
\text { Fiorito, } \\
1994(32)\end{array}$ & $\begin{array}{l}425 \text { nurses at a general } \\
\text { hospital and } 198 \text { nurses } \\
\text { in a small oncology } \\
\text { ward, Italy }\end{array}$ & $\begin{array}{l}\text { Medical examination S, PSE } \\
\text { with an interview; } \\
\text { Selected cases had } \\
\text { X-ray examination } \\
\text { or orthopedic } \\
\text { examination }\end{array}$ & $\begin{array}{l}\text { Two hospitals with } \\
\text { different organiza- } \\
\text { tion }\end{array}$ \\
\hline
\end{tabular}

LBP during the last Lifting heavy objects, $O R=2.81$ year $\quad(95 \% \mathrm{Cl} 1.88-4.20)$; work experience, $\mathrm{OR}=2.33(95 \% \mathrm{Cl} 1.53-$ $3.57)$; sitting habits, $\mathrm{OR}=2.13$ (95\% Cl $1.37-2.80)$

Back pain in past a) $O R=1, b) O R=1.50(P<0.01)$, 12 months $\quad$ c) $0 R=2.25(P<0.001)$; nurses: aides or registered nurses, $\mathrm{OR}=1.32$ $(P=0.10)$; NS: home-work commute, number of years in occupation $\begin{array}{ll}\text { Nurses being } & \text { Prior nonback injury, } O R=3.66 \\ \text { awarded a claim for }(95 \% \mathrm{Cl} 1.52-8.83) ; \text { twisting and }\end{array}$ work-related back lifting, $O R=4.84(95 \% \mathrm{Cl} 1.30-$ injury; occupational 18.04); NS: age, gender, job title, back injury history overweight

Musculoskeletal Differences between general hospisymptoms; work- tal and oncology ward: LBP due to related injuries, ab- work during last year $48 / 33 \%$ sence due to illness $(P<0.001)$, ratio of nurse to patient $0.57 / 1.27$, NS: length of employment; lifting was the factor that most nurses related to back injury (50\%), bending $(42 \%)$, and twisting $(30 \%)$ $(95 \%$ Cl $1.46-3.11)$; age, $\mathrm{OR}=1.96$ 
Table 1. Continued

\begin{tabular}{|c|c|c|c|c|c|c|}
\hline Reterence & Population & $\begin{array}{l}\text { Data } \\
\text { collection }\end{array}$ & $\begin{array}{l}\text { Quality } \\
\text { comments }\end{array}$ & $\begin{array}{l}\text { Work-related } \\
\text { risk factors }\end{array}$ & $\begin{array}{l}\text { Outcome } \\
\text { measure }\end{array}$ & Main findings \\
\hline $\begin{array}{l}\text { Burton et al, } \\
1997(33)\end{array}$ & $\begin{array}{l}\text { 1, } 1216 \text { nurses from } 7 \\
\text { hospitals in Belgium } \\
\text { and The Netherlands }\end{array}$ & Questionnaire & $S, P R$ & $\begin{array}{l}\text { Psychosocial fac- } \\
\text { tors, work load } \\
\text { (ie, time spent in } \\
\text { nursing activities) }\end{array}$ & $\begin{array}{l}\text { Current back prob- } \\
\text { lems, history of LBP }\end{array}$ & $\begin{array}{l}\text { Prevalence of LBP was not depend- } \\
\text { ent on work load; prevalence rates } \\
\text { for LBP higher for Belgian nurses, } \\
\text { higher work load and more positive } \\
\text { attitudes toward work among } \\
\text { Dutch nurses }\end{array}$ \\
\hline $\begin{array}{l}\text { Lagerström } \\
\text { et al, } 1995 \\
\text { (13) }\end{array}$ & $\begin{array}{l}688 \text { female nursing } \\
\text { persomel at } 1 \text { hospital } \\
\text { in Sweden; registered } \\
\text { nurses, state enrolled } \\
\text { nurses, and auxiliary } \\
\text { nurses }\end{array}$ & Questionnaire & PhE & $\begin{array}{l}\text { Physical exposure } \\
\text { at work and psycho- } \\
\text { social factors at } \\
\text { work }\end{array}$ & $\begin{array}{l}\text { Ongoing LBP di- } \\
\text { chotomized into } \\
\text { symptoms or } \\
\text { severe symptoms }\end{array}$ & $\begin{array}{l}\text { Symptoms: OR=1.80 (95\% Cl } \\
1.13-2.86 \text { ) for low support at } \\
\text { work, OR=1.79 ( } 95 \% \mathrm{Cl} 1.26- \\
2.54 \text { ) for low physical fitness, } \\
\text { OR=1.70 (95\% Cl } 1.13-2.56) \text { for } \\
\text { work category (registered } \\
\text { nurse / auxiliary nurse); severe } \\
\text { symptoms: OR=1.60 (95\% Cl } \\
1.04-2.46) \text { for low physical } \\
\text { fitness, NS for age, body mass } \\
\text { index, smoking, work commitment, } \\
\text { work demand, skill utilization, job } \\
\text { control }\end{array}$ \\
\hline
\end{tabular}

Stobbe et 143 licensed practi- Discussions with PSE $\quad$ a) High lifting tre- Back injuries during IR of back injuries by frequency of

al, 1988 cal nurses, 252

(34) [in-

cluded in attendants at 28

$(20)] \quad$ wards in one hospital

nursing super-

visors, register

data from the

in the United States

Owen,

32 nurses with back

hospital

Questionnaire,

PR

injuries and 32 referents interview and phys

from a university ical tests and acci-

hospital in the United dent reports

States

Bru et al, 586 female health care

Questionnaire

PhE

Psychosocial and

LBP

workers at 1 regional

hospital in Norway factors

Rydén et 84 cases with low-back Data obtained from $S$, PhE, PSE Records of the peral, 1989 injuries during 4 years (9) and 168 matched the Employee Health Service in San Diego, United States

Ljungberg 24 nurses' aides from 2 Observation LBP

(2)

$$
\begin{aligned}
& \text { geriatric care units in (OWAS), health } \\
& \text { Stockholm, a modern check with muscle } \\
& \text { ward with team-care and strength, patient } \\
& \text { a ward with traditional capability scores, }
\end{aligned}
$$
care of nursing official records

Skrovdon 787 nursing staff at 2 et al, 1987 hospitals in New York (39) City, United States
Questionnaire

PhE, PR Work organization

factors, mode of nursing care, frequency of lifting.

satisfaction with perceived autonomy, so cial support at work work organizational

40 months (report - lifting exposure $(a / b): I R=754$ for

ed by 23 licensed licensed practical nurses, $I R=2.11$ practical nurses, for nurses' aides, $I R=1.07$ for 44 nurses' aides, attendants; length of employment and 5 attendants) and lifting frequency related to back injuries

Back injuries report- Most important factors related to ed in accident LBP in multivariate analyses: a sense of overload, muscle flexibility, proprioception, family history of LBP, difference in leg length, years of risk in nursing practice, and smoking

Age, work overload, institutional policy, social relations at work related to LBP among nurses with high ergonomic load; responsibility related to LBP among nurses with low ergonomic load

Reported low-back History of previous back injury at injuries (to Emp- work, $\mathrm{OR}=2.13(95 \% \mathrm{Cl} 1.07$ loyee Health $\quad 4.24$ ); workday shift, $\mathrm{OR}=2.23$ Service) (95\% Cl $1.28-3.89) ; 55 \%$ of injuries from lifting; NS: history of previous injury at work, working evening or night shift, smoking itts, space, work juries of the back load, mode of nurs- per 1000 employed ing care during 4 years

modern ward (mode of nursing

care; traditional/ team-care), number of back injuries per 1000 nurses: 50 and 25; mean age: 35 and 24 years $(P<0.05)$; total number of lifts per hour: 30 and 14 $(P<0.01)$; total time for lifts in seconds per hour: 309 and 68 $(P<0.001)$; NS: total number of steps per hour and percentage of time in static posture Younger age ( $P=0.03$ ), job category "registered nurse" $(P=0.03)$, great satisfaction with co-workers (!) $(P=0.05)$, NS: factors within the work organization, support from head nurse, perceived autonomy of job, mode of nursing care 
Table 1. Continued

\begin{tabular}{|c|c|c|c|c|c|c|}
\hline Reference & Population & $\begin{array}{l}\text { Data } \\
\text { collection }\end{array}$ & $\begin{array}{l}\text { Quality } \\
\text { comments }\end{array}$ & $\begin{array}{l}\text { Work-related } \\
\text { risk factors }\end{array}$ & $\begin{array}{l}\text { Outcome } \\
\text { measure }\end{array}$ & Main findings \\
\hline $\begin{array}{l}\text { Lo et al } \\
1993(41)\end{array}$ & $\begin{array}{l}37 \text { female nurses in a } \\
\text { geriatric hospital in } \\
\text { West Australia }\end{array}$ & $\begin{array}{l}\text { Diaries and survey } \\
\text { sheets during } 7 \\
\text { days, questionnaire }\end{array}$ & PSE, LBP & $\begin{array}{l}\text { Patient lifts: point } \\
\text { of time, type, train- } \\
\text { ing in transfer tech- } \\
\text { nique, and data of } \\
\text { the patients (weight, } \\
\text { etc) }\end{array}$ & $\begin{array}{l}\text { Work-related back } \\
\text { problems }\end{array}$ & $\begin{array}{l}\text { Total weight lifted not in accord- } \\
\text { ance with any of the recommended } \\
\text { methods as the main risk factor: } \\
0 R=1.07 \text { ( } 95 \% \mathrm{Cl} 1.00-1.21 \text { ) for } \\
\text { each additional } 100 \mathrm{~kg} \text { lifted: } \\
\text { factors contributing to choice of } \\
\text { method were patient co-operation, } \\
\text { occupational category and number } \\
\text { of nurses carrying out the lift }\end{array}$ \\
\hline $\begin{array}{l}\text { Ahlberg- } \\
\text { Hultén et } \\
\text { al, } 1995 \\
\text { (43) }\end{array}$ & $\begin{array}{l}90 \text { female nurses and } \\
\text { nurses aides from } \\
\text { different types of wards } \\
\text { in different medium- } \\
\text { sized hospitals in the } \\
\text { Stockholm area }\end{array}$ & $\begin{array}{l}\text { Data from } 2 \text { studies: } \\
\text { group discussions, } \\
\text { questionnaire }\end{array}$ & PhE & $\begin{array}{l}\text { Psychological de- } \\
\text { mands, authority } \\
\text { over decisions, skill } \\
\text { utilization (ie, job } \\
\text { strain ratio), social } \\
\text { support at work, and } \\
\text { physical strain }\end{array}$ & $\begin{array}{l}\text { LBP sometimes or } \\
\text { daily }\end{array}$ & $\begin{array}{l}\text { Job strain, high/low } 0 R=2.00 \text { ( } 95 \% \\
\text { Cl } 1.44-2.25) \text {; factors related to } \\
\text { LBP for both nurses and nurses } \\
\text { aides were high psychological } \\
\text { demands ( } P=0.03) \text {, low authority } \\
\text { over decisions }(P=0.05) \text {, low skill } \\
\text { utilization ( } P=0.05)\end{array}$ \\
\hline $\begin{array}{l}\text { Nied- } \\
\text { hammer } \\
\text { et al, } 1994 \\
\text { (44) }\end{array}$ & $\begin{array}{l}469 \text { nurses in } 10 \\
\text { French hospitals }\end{array}$ & $\begin{array}{l}\text { Questionnaire, inter- } \\
\text { view (for nurses } \\
\text { who had left work) } \\
\text { (cross-sectional, the } \\
\text { prospective cohort } \\
\text { study } 1980-1990 ; \\
\text { see table } 4 \text { ) }\end{array}$ & S, PR & $\begin{array}{l}\text { Work description, } \\
\text { work conditions, } \\
\text { work organization, } \\
\text { shift work }\end{array}$ & $\begin{array}{l}\text { LBP in previous } \\
12 \text { months and } \\
\text { musculoskeletal } \\
\text { disorders }\end{array}$ & $\begin{array}{l}\text { Age of } 40-44 /<34 \text { years, } O R= \\
2.05(P=0.09) ; 3 \text { or more } \\
\text { symptoms of psychological } \\
\text { disorders/none, } 0 R=2.64(P=0.01) \\
\text { physical work load/none, } O R=2.23 \\
(P=0.04) \text {; NS: smoking, number of } \\
\text { children, sports activities, previous } \\
\text { musculoskeletal disorders, psycho- } \\
\text { social factors at work }\end{array}$ \\
\hline
\end{tabular}

a 1.6 million women.

Table 2. Work-related risk factors for low-back problems in nursing - prospective cohort studies. (NS= not significant; incomplete information: $\mathrm{S}=$ sample, $\mathrm{PhE}=$ physical exposure, $\mathrm{PSE}=$ psychosocial exposure, $\mathrm{LBP}=$ low back problems, $\mathrm{PR}=$ participation rate $<70 \%$; $\mathrm{FR}=$ follow-up rate $<70 \%$ )

\begin{tabular}{|c|c|c|c|c|c|c|}
\hline Reference & Follow-up length & $\begin{array}{l}\text { Population and } \\
\text { data collection }\end{array}$ & $\begin{array}{l}\text { Quality } \\
\text { comments }\end{array}$ & $\begin{array}{l}\text { Work-related } \\
\text { risk factors }\end{array}$ & $\begin{array}{l}\text { Outcome } \\
\text { measure }\end{array}$ & Main findings and conclusions \\
\hline $\begin{array}{l}\text { Smedley et } \\
\text { al, } 1997 \\
(22)\end{array}$ & 2-year follow-up & $\begin{array}{l}\text { Of } 1336 \text { nurses } 961 \\
\text { nurses without LBP } \\
\text { the last month in } \\
\text { the United Kindom; } \\
\text { questionnaire }\end{array}$ & PSE & $\begin{array}{l}\text { Frequency of } \\
\text { occupational } \\
\text { activities }\end{array}$ & $\begin{array}{l}\text { (a) incidence of all } \\
\text { LBP, (b) LBP } \\
\text { leading to absence } \\
\text { from work }\end{array}$ & $\begin{array}{l}\text { Transfer of patients between bed } \\
\text { and chair manually } 5-9 \text { times per } \\
\text { shift (a) OR=1.6 ( } 95 \% \mathrm{Cl} 1.1-2.3 \text {, } \\
\text { (b) } \mathrm{OR}=1.9(95 \% \mathrm{Cl} 1.0-3.7) \text {; } \\
\text { transfer of patient between bed and } \\
\text { chair with hoist }>5 \text { times (a) NS, (b) } \\
\text { NS; lift patients in or out of bath } \\
\text { manually }>1 \text { time per shift (a) } \\
\text { OR=NS, (b) NS; lifting patient in or } \\
\text { out of bath with hoist }>1 \text { time per } \\
\text { shift (a) OR=2.1 (95\% Cl 1.2-3.6, } \\
\text { (b) NS (adjusted for age, height and } \\
\text { earlier history of back problems) }\end{array}$ \\
\hline $\begin{array}{l}\text { Venning ef } \\
\text { al, } 1987 \\
(23) ; \mathrm{a} \\
\text { [included in } \\
(20) \text { ] }\end{array}$ & 12-month follow-up & $\begin{array}{l}4024 \text { nurses aides, } \\
\text { orderlies and } \\
\text { registered nurses } \\
\text { from } 5 \text { hospitals in } \\
\text { Canada; question- } \\
\text { naire }\end{array}$ & PsE & $\begin{array}{l}\text { Ergonomic factors: } \\
\text { work category, lift- } \\
\text { ing requirements, } \\
\text { service area, hours } \\
\text { and type of shift, } \\
\text { length of employ- } \\
\text { ment, training in } \\
\text { back care and lifting, } \\
\text { team lifting, avail- } \\
\text { ability of lifting aids } \\
\text { and of light duty }\end{array}$ & $\begin{array}{l}\text { Report on back } \\
\text { injury during } \\
\text { tollow-up period }\end{array}$ & $\begin{array}{l}\text { Service areas with the most or least } \\
\text { lifting } O R=4.26(P<.01) \text {; lifters daily } \\
\text { or other } 0 R=2.19(P<.05) \text {; nurses' } \\
\text { aids or registered nurses and } \\
\text { supervisory personnel } O R=1.77 \\
\text { ( } P<.01) \text {; previously reported back } \\
\text { injury yes or no } O R=1.73(P<.01) \text {; } \\
\text { NS: availability of lifting devices and } \\
\text { training in back care and lifting }\end{array}$ \\
\hline $\begin{array}{l}\text { Heap, } 1987 \\
(35)\end{array}$ & 5-year follow-up & $\begin{array}{l}3778 \text { nursing staff } \\
\text { with } 180 \text { low-back } \\
\text { injuries in the } \\
\text { United Kingdom, } \\
\text { clinical examination } \\
\text { with an interview }\end{array}$ & PSE & $\begin{array}{l}\text { Nursing procedures } \\
\text { and patient handling } \\
\text { maneuver, type of } \\
\text { lift, staffing level, } \\
\text { and other ergonomic } \\
\text { factors }\end{array}$ & $\begin{array}{l}\text { Injuries in the low } \\
\text { back reported on } \\
\text { standard accident } \\
\text { forms }\end{array}$ & $\begin{array}{l}\text { Previous significant back injury } \\
\text { predicted subsequent low-back } \\
\text { injury; higher injury rate among } \\
\text { nurses' aides than among } \\
\text { registered nurses }\end{array}$ \\
\hline
\end{tabular}


Table 2. Continued

\begin{tabular}{|c|c|c|c|c|c|c|}
\hline Reference & Follown up length & $\begin{array}{l}\text { Population and } \\
\text { data collection }\end{array}$ & $\begin{array}{l}\text { Quality } \\
\text { comments }\end{array}$ & $\begin{array}{l}\text { Work-related } \\
\text { risk factors }\end{array}$ & $\begin{array}{l}\text { Outcome } \\
\text { measure }\end{array}$ & Main findings and conclusions \\
\hline $\begin{array}{l}\text { Yassi et al, } \\
1995(36)\end{array}$ & 2-year follow-up & $\begin{array}{l}1645 \text { nurses with } \\
416 \text { back injuries, } \\
\text { Canada }\end{array}$ & $S, P S E$ & $\begin{array}{l}\text { Occupational } \\
\text { history, mechanics } \\
\text { and circumstances } \\
\text { of injury, and ergo- } \\
\text { nomic factors }\end{array}$ & $\begin{array}{l}\text { Back injury reports } \\
\text { filled out by the } 416 \\
\text { persons with back } \\
\text { injuries }\end{array}$ & $\begin{array}{l}\text { When, where, how and why injuries } \\
\text { occurred: work in orthopedic wards } \\
(51 \%) \text {; assisted lifts and transfers } \\
(46 \%) \text { and unassisted lifts }(11 \%) \\
\text { "save the patient" situations }(7 \%) \text {; } \\
\text { inadequate training ( } 52 \%) \text { and } \\
\text { staffing ( } 14 \%) ; 2 \text { first hours of } \\
\text { 8-hour shift ( } 53 \%)\end{array}$ \\
\hline $\begin{array}{l}\text { Harber et al, } \\
1994(40)^{\mathrm{a}}\end{array}$ & $\begin{array}{l}\text { 18-month study with an } \\
\text { average of } 10 \text {-months of } \\
\text { follow-up }\end{array}$ & $\begin{array}{l}179 \text { nurses newly } \\
\text { graduated from } \\
\text { nursing school in } \\
\text { the United States; } \\
\text { telephone interviews } \\
\text { and reinterviews } \\
\text { (pilot study) }\end{array}$ & S, PR, FR & $\begin{array}{l}\text { Work site factors; } \\
\text { shift length, patient } \\
\text { type, availability of } \\
\text { mechanical lifting } \\
\text { devices and human } \\
\text { help in lifting; psy- } \\
\text { chosocial factors } \\
\text { and training }\end{array}$ & $\begin{array}{l}\text { Each subject classi- } \\
\text { fied as an injured or } \\
\text { a noninjured } \\
\text { subject based on } \\
\text { responses to } \\
\text { questions about } \\
\text { back problems; } \\
\text { prior back problems }\end{array}$ & $\begin{array}{l}\text { Previous significant back problem } \\
\text { episodes associated with increased } \\
\text { future risk; NS: training at nursing } \\
\text { school }\end{array}$ \\
\hline $\begin{array}{l}\text { Josephson } \\
\text { et al, } 1997 \\
(42)\end{array}$ & $\begin{array}{l}\text { Base line and follow-up } \\
\text { once a year during } 3 \\
\text { years }\end{array}$ & $\begin{array}{l}285 \text { female health } \\
\text { care workers at one } \\
\text { hospital in Sweden; } \\
\text { questionnaire }\end{array}$ & PhE, FR & $\begin{array}{l}\text { Perceived physical } \\
\text { exertion; job strain } \\
\text { (combination of } \\
\text { high mental } \\
\text { demands and low } \\
\text { decision latitude) }\end{array}$ & $\begin{array}{l}\text { Neck, shoulder or } \\
\text { low-back problems }\end{array}$ & $\begin{array}{l}\text { High psychological demands, low } \\
\text { decision latitude, and perceived } \\
\text { physical exertion on the same mea- } \\
\text { sure occasion } R R=1.5(95 \% \mathrm{Cl} \\
1.1-2.1)-2.1 \text { ( } 95 \% \mathrm{Cl} 1.4- \\
3.3) \text {; high psychological demands, } \\
\text { low decision latitude } 1.2 \text { or } 3 \text { years } \\
\text { later } R R=1.4(95 \% \mathrm{Cl} 0.5-4.0)- \\
2.2(95 \% \mathrm{Cl} 0.8-6.0) \text { for having } \\
\text { musculoskeletal symptoms }\end{array}$ \\
\hline $\begin{array}{l}\text { Nied- } \\
\text { hammer et } \\
\text { al, } 1994 \\
(44)\end{array}$ & 10-year & $\begin{array}{l}300 \text { nurses in } 6 \\
\text { public sector hospi- } \\
\text { tals in France; ques- } \\
\text { tionnaire (interview } \\
\text { for nurses who had } \\
\text { left work) }\end{array}$ & $\mathrm{S}, \mathrm{FR}$ & $\begin{array}{l}\text { Work description, } \\
\text { work conditions, } \\
\text { work organization, } \\
\text { and shift work }\end{array}$ & $\begin{array}{l}\text { LBP in previous } 12 \\
\text { months and } \\
\text { musculoskeletal } \\
\text { disorders }\end{array}$ & $\begin{array}{l}\text { Commuting }>1 /<1 \text { hour a day } \\
\text { OR= } 2.00 \text { ( } P=0.008) ; \text { NS: age, } \\
\text { psychological disorders, physical } \\
\text { work load, smoking, number of } \\
\text { children, sports activities, previous } \\
\text { musculoskeletal disorders, and } \\
\text { psychosocial factors at work }\end{array}$ \\
\hline $\begin{array}{l}\text { Ready et al } \\
1993(45)^{\mathrm{a}}\end{array}$ & 18*month follow-up & $\begin{array}{l}131 \text { nurses and unit } \\
\text { assistants at a large } \\
\text { health care center in } \\
\text { Canada; question- } \\
\text { naire, fitness as- } \\
\text { sessment }\end{array}$ & $\begin{array}{l}\text { S, PhE, } \\
\text { PSE, PR }\end{array}$ & $\begin{array}{l}\text { Wards: high, medi- } \\
\text { um, or low risk, em- } \\
\text { ployment history }\end{array}$ & $\begin{array}{l}\text { Back injuries re- } \\
\text { ported by subjects } \\
\text { on an employee ac- } \\
\text { cident report, his- } \\
\text { tory of back injury }\end{array}$ & $\begin{array}{l}\text { Prior compensation pay, smoking } \\
\text { status, and job satisfaction were the } \\
\text { most important factors for back } \\
\text { injuries, NS: fitness, life-style } \\
\text { factors } \\
\text { Attitudes towards health, personal }\end{array}$ \\
\hline $\begin{array}{l}\text { Klaber } \\
\text { Moffett et } \\
\text { al, } 1993 \\
(46)\end{array}$ & 30-month follow-up & $\begin{array}{l}199 \text { student nurses } \\
\text { on entry to } 2 \\
\text { schools of nursing } \\
\text { in the United King- } \\
\text { dom; physical tests } \\
\text { of strength endur- } \\
\text { ance of the muscles; } \\
\text { questionnaire, dia- } \\
\text { ries with recordings } \\
\text { of LBP }\end{array}$ & $\begin{array}{l}\text { PhE, PSE, } \\
\text { FR }\end{array}$ & $\begin{array}{l}\text { Initial screening for } \\
\text { physical and psy- } \\
\text { chosocial factors; } \\
\text { follow-up every 3rd } \\
\text { month to collect } \\
\text { diaries with record- } \\
\text { ings of problems, } \\
\text { questionnaire, tests } \\
\text { of strength endur- } \\
\text { ance of the muscles }\end{array}$ & $\begin{array}{l}\text { Back problems } \\
\text { during at least } 3 \\
\text { days (group 1) or } \\
\text { back problems } \\
\text { during } 21 \text { days } \\
\text { (group 2) }\end{array}$ & $\begin{array}{l}\text { psychology factors, strength } \\
\text { endurance of the thigh muscles, } \\
\text { and height predicted (group } 1 \text { and } \\
2 \text {, height not group 2) }\end{array}$ \\
\hline $\begin{array}{l}\text { Mostardi } \\
\text { et al, } 1992 \\
(52)^{\mathrm{a}}\end{array}$ & 2-year follow-up & $\begin{array}{l}171 \text { female nurses } \\
\text { in a tertiary care } \\
\text { community hospital } \\
\text { in Ohio; isokinetic } \\
\text { lifting test, } \\
\text { questionnaire, injury } \\
\text { reports }\end{array}$ & S, PSE & $\begin{array}{l}\text { Isokinetic lifting } \\
\text { test, medical history } \\
\text { and individual data }\end{array}$ & $\begin{array}{l}\text { Injury reports of } \\
\text { job-related LBP or } \\
\text { injury during the } \\
\text { 2-year follow-up } \\
\text { period, medical } \\
\text { history }\end{array}$ & previous significant back injury \\
\hline
\end{tabular}

a Studies included in Bigos et al, 1996 (19).

Questionnaire (18)] or secondary data (ie, register data on injury reports).

4. In studies on nursing work, participation rate is often a problem.

The intervention studies were further reviewed for the following methodological quality issues (see table 3):

5. Nonrandom assignment of subjects, as random assignment prevents selection bias.
6. Lack of a reference group, as the inclusion of a reference group makes it possible to separate the effects of the intervention and the effects of confounders.

7. Description of the intervention not informative enough for other researchers to replicate the original research.

A special committee was appointed in 1995 by the American Academy of Orthopaedic Surgeons (AAOS) to clarify the scientifically proved basis of knowledge 
Table 3. Prevention of low back problems in nursing - intervention studies. $(I=$ individual level of intervention; $0=$ organizational leve of intervention; incomplete information: $\mathrm{S}=$ sample, $\mathrm{PhE}=$ physical exposure, $\mathrm{PSE}=$ psychosocial exposure, $\mathrm{LBP}=$ low-back problems IV = intervention, $P R=$ participation rate $<70 \% ; F R=$ follow-up rate $<70 \% ; R=$ randomization not performed, $N R=$ no reference group)

\begin{tabular}{|c|c|c|c|c|c|c|}
\hline Reference & Follow-up length & $\begin{array}{l}\text { Population and } \\
\text { data collection }\end{array}$ & $\begin{array}{l}\text { Quality } \\
\text { comments }\end{array}$ & $\begin{array}{l}\text { Intervention } \\
\text { studied }\end{array}$ & $\begin{array}{l}\text { Outcome } \\
\text { measure }\end{array}$ & Main findings \\
\hline $\begin{array}{l}\text { Gundewall } \\
\text { et al, } 1993 \\
(54)^{\mathrm{a}}\end{array}$ & 13 months & $\begin{array}{l}32 \text { nurses aides } \\
\text { (and } 28 \text { referents) } \\
\text { with and without } \\
\text { history of LBP in a } \\
\text { geriatric hospital } \\
\text { questionnaire; } \\
\text { report card, } \\
\text { physical tests }\end{array}$ & PhE, PsE & $\begin{array}{l}\text { Group A (training): } \\
\text { instructions on } \\
\text { exercises for } \\
\text { strengthening back } \\
\text { extensors provided } \\
\text { during 5.5-hour } \\
\text { sessions with } \\
\text { individual instruc- } \\
\text { tions followed by } 13 \\
\text { months of exercise } \\
\text { sessions at work; } \\
\text { group B (reference): } \\
\text { no exercise or } \\
\text { training sessions } \\
\text { provided }\end{array}$ & $\begin{array}{l}\text { Isometric back } \\
\text { muscle strength, } \\
\text { days experiencing } \\
\text { LBP, days absent } \\
\text { from work because } \\
\text { of LBP; level I }\end{array}$ & $\begin{array}{l}\text { Intervention subjects demonstrated } \\
\text { greater extensor strength than ref- } \\
\text { erents, less absenteeism due to } \\
\text { LBP during } 13 \text { months ( } 1.0 / 4.8 \\
\text { days) and fewer days with LBP ( } 54 \\
\text { / } 94 \text { days); every hour spent by the } \\
\text { physiotherapist on the training } \\
\text { group reduced the work absence } \\
\text { among the participants by } 1.3 \text { days, } \\
\text { resulting in a cost-benefit ratio of } \\
>10\end{array}$ \\
\hline $\begin{array}{l}\text { Videman } \\
\text { et al, } 1989 \\
(55)\end{array}$ & 2.5 years & $\begin{array}{l}87 \text { student nurses } \\
\text { with extra training } \\
\text { and } 113 \text { student } \\
\text { nurses without } \\
\text { extra training } \\
\text { (referents), } \\
\text { questionnaire, anth- } \\
\text { ropometric mea- } \\
\text { surements, strength } \\
\text { and functional tests }\end{array}$ & $\begin{array}{l}\text { R, PhE, } \\
\text { PSE }\end{array}$ & $\begin{array}{l}\text { Referents started } 1 \\
\text { course and received } \\
\text { traditional training in } \\
\text { patient handling; } \\
\text { students who started } \\
\text { next year got special } \\
40 \text { hours of training } \\
\text { based on ergonomic } \\
\text { principles }\end{array}$ & $\begin{array}{l}\text { Isometric strength, } \\
\text { psychological } \\
\text { factors and back } \\
\text { problems; level I }\end{array}$ & $\begin{array}{l}\text { The subsequent incidence of acute } \\
\text { back injuries occurring at work was } \\
\text { significantly lower among the } \\
\text { skilled nurses but there was no } \\
\text { difference between skilled and } \\
\text { unskilled nurses in the incidence of } \\
\text { other forms of back problems }\end{array}$ \\
\hline $\begin{array}{l}\text { Hellsing } \\
\text { et al, } 1993 \\
(56)\end{array}$ & 2 years & $\begin{array}{l}2 \text { nursing schools: } \\
19 \text { student nurses } \\
\text { with extra education } \\
\text { of } 2 \text { hours per week } \\
\text { during } 2 \text { years and } \\
33 \text { student nurses } \\
\text { who received the } \\
\text { usual curriculum } \\
\text { were referents; } \\
\text { questionnaire }\end{array}$ & $R$ & $\begin{array}{l}\text { Extra education } \\
\text { included theory and } \\
\text { practice relaxation } \\
\text { and physical, } \\
\text { psychological and } \\
\text { ergonomic training }\end{array}$ & $\begin{array}{l}\text { Evaluation of the } \\
\text { extra education, } \\
\text { neck-shoulder and } \\
\text { LBP; level I }\end{array}$ & $\begin{array}{l}\text { No obvious short-time effect of the } \\
\text { education on musculoskeletal } \\
\text { problems (low back) could be seen; } \\
\text { the experimental group judged their } \\
\text { ability to analyze the work } \\
\text { environment higher than the } \\
\text { referents: observations showed that } \\
\text { students from the experience group } \\
\text { worked in physically more favorable } \\
\text { positions }\end{array}$ \\
\hline $\begin{array}{l}\text { Lageström } \\
\text { et al, } 1998 \\
(57)\end{array}$ & $\begin{array}{l}3 \text { years intervention } \\
\text { with } 1 \text { year follow-up }\end{array}$ & $\begin{array}{l}348 \text { registered } \\
\text { nurses, state } \\
\text { enrolled nurses, } \\
\text { and nursing aides; } \\
\text { questionnaire }\end{array}$ & $R, C, F R$ & $\begin{array}{l}\text { Training and } \\
\text { education in patient } \\
\text { transfer technique, } \\
\text { stress handling, and } \\
\text { physical fitness } \\
\text { exercise }\end{array}$ & $\begin{array}{l}\text { Work and leisure- } \\
\text { time factors, } \\
\text { physical exertion, } \\
\text { and musculoskeletal } \\
\text { problems; } \\
\text { level I }\end{array}$ & $\begin{array}{l}93 \% \text { of the nurses used the new } \\
\text { work technique always or mostly; } \\
\text { nurses who did not use the work } \\
\text { I technique rated some transfers as } \\
\text { physically more strenuous than } \\
\text { those who used the technique; in- } \\
\text { creased rate of nurses transferred } \\
\text { patients alone when } 2 \text { were needed; } \\
\text { no decrease in LBP }\end{array}$ \\
\hline $\begin{array}{l}\text { Feldstein } \\
\text { et al, } 1993 \\
(58)\end{array}$ & $\begin{array}{l}\text { 1-month follow-up; } \\
\text { pilot study }\end{array}$ & $\begin{array}{l}30 \text { nurses (and } 25 \\
\text { referents); ques- } \\
\text { tionnaire, transfer } \\
\text { forms, physical } \\
\text { tests }\end{array}$ & $R, P R$ & $\begin{array}{l}\text { Training in lifting } \\
\text { technique and } \\
\text { muscle strengthen- } \\
\text { ing training }\end{array}$ & $\begin{array}{l}\text { Back problems; } \\
\text { technique when } \\
\text { transferring pa- } \\
\text { tients, muscle } \\
\text { flexibility, proprio- } \\
\text { ceptive ability; } \\
\text { level I }\end{array}$ & $\begin{array}{l}\text { No difference in back problems } \\
\text { between the intervention and } \\
\text { reference groups; higher quality of } \\
\text { patient transfer in the intervention } \\
\text { group }\end{array}$ \\
\hline $\begin{array}{l}\text { Garg \& } \\
\text { Owen, } 1992 \\
(59)\end{array}$ & $2^{8 \text { months }}$ & $\begin{array}{l}38 \text { nursing } \\
\text { assistants at a } \\
\text { nursing home in } \\
\text { Wisconsin, United } \\
\text { States; observation } \\
\text { (data collection } \\
\text { forms), Borg's } \\
\text { rating of percieved } \\
\text { exertion, accident } \\
\text { reports, biome- } \\
\text { chanical test }\end{array}$ & C, PsE, FR & $\begin{array}{l}\text { Modified toilets, } \\
\text { showers and other } \\
\text { devices; ergonomic } \\
\text { training to use the } \\
\text { devices; teaching } \\
\text { and training in } \\
\text { patient transfer } \\
\text { technique }\end{array}$ & $\begin{array}{l}\text { a) incidence rate of } \\
\text { back problems, b) } \\
\text { compressive force } \\
\text { on the L5/S1 disc, } \\
\text { c) hand force, d) } \\
\text { percentage of } \\
\text { nurses' aides capa- } \\
\text { ble of performing } \\
\text { stressful patient- } \\
\text { handling tasks, e) } \\
\text { perceived physical } \\
\text { exertion; level I + } 0\end{array}$ & $\begin{array}{l}\text { Before and after measurements: a) } \\
83 \& 47 / 200000 \text { workhours, b) } \\
4751 \mathrm{~N} / 1964 \mathrm{~N}, \mathrm{c}) 312 \mathrm{~N} / 122 \mathrm{~N} \text {, } \\
\text { d) } 41 / 83 \% \text {, e) somewhat hard and } \\
\text { hard or very light }\end{array}$ \\
\hline
\end{tabular}


Table 3. Contiunued

\begin{tabular}{|c|c|c|c|c|c|c|}
\hline Reference & Follow-up length & $\begin{array}{l}\text { Population and } \\
\text { data collection }\end{array}$ & $\begin{array}{l}\text { Quality } \\
\text { comments }\end{array}$ & $\begin{array}{l}\text { intervention } \\
\text { studied }\end{array}$ & $\begin{array}{l}\text { Outcome } \\
\text { measure }\end{array}$ & Main findings \\
\hline $\begin{array}{l}\text { Yassi et al, } \\
1995(60)\end{array}$ & $\begin{array}{l}6 \text { months of follow-up } \\
\text { after return to work }\end{array}$ & $\begin{array}{l}250 \text { nurses with } \\
\text { back problems } \\
\text { (experiment group) } \\
\text { and } 1395 \text { referents } \\
\text { at the same hospital; } \\
\text { interview }\end{array}$ & $\begin{array}{l}S, R, P R \& \\
\text { FR no info }\end{array}$ & $\begin{array}{l}\text { Assessment } \\
\text { treatment by a } \\
\text { physiotherapist; if } \\
\text { sick leave }>4 \text { days } \\
\text { occupational therapy } \\
\text { assessment; early } \\
\text { return to work with } \\
\text { individually modified } \\
\text { work tasks; referents } \\
\text { asked to seek care } \\
\text { elsewhere }\end{array}$ & $\begin{array}{l}\text { Incidence of back } \\
\text { problems and } \\
\text { absence, costs for } \\
\text { sick leave because } \\
\text { of back problems; } \\
\text { level I+0 } \\
\end{array}$ & $\begin{array}{l}\text { The number of reported back in- } \\
\text { juries among the nurses dropped } \\
\text { and absence due to back problems } \\
\text { decreased in the experimental } \\
\text { group; in the reference group there } \\
\text { was an increase in these factors; the } \\
\text { costs for back injuries and absence } \\
\text { due to this were decreased by } 23 \% \\
\text { and } 43 \% \text {, respectively, in the wards } \\
\text { where the experimental group work- } \\
\text { ed, while the costs for the reference } \\
\text { group increased }\end{array}$ \\
\hline $\begin{array}{l}\text { Wood, } \\
1987(61)\end{array}$ & 1 year & $\begin{array}{l}\text { Employees at } 1 \\
\text { hospital which con- } \\
\text { sisted of } 3 \text { units } \\
\text { ( } 2 \text { intervention and } \\
1 \text { referent); accident } \\
\text { reports }\end{array}$ & $\begin{array}{l}\text { S, R, PSE; } \\
\text { PR \& FR no } \\
\text { info }\end{array}$ & $\begin{array}{l}\text { a) personnel pro- } \\
\text { gram: increase con- } \\
\text { tact with newly injur-- } \\
\text { ed nurse and hospit- } \\
\text { al staff and doctor so } \\
\text { that the injured } \\
\text { nurse would not be } \\
\text { lost in the system; b) } \\
\text { back program: } \\
\text { evaluate ability to } \\
\text { transfer patients in a } \\
\text { classroom and at } \\
\text { own wards, training } \\
\text { in how to use }\end{array}$ & $\begin{array}{l}\text { Wage-loss claims } \\
\text { filed by nurses for } \\
\text { - back injuries caused } \\
\text { by interaction with } \\
\text { o residents; level I } \\
\text { (back program) + } 0 \\
\text { (personnel pro- } \\
\text { gram) }\end{array}$ & $\begin{array}{l}\text { The back program, training in } \\
\text { patient transfer technique, did not } \\
\text { reduce the back injuries; the } \\
\text { personnel program reduced the } \\
\text { back injuries; the combination of } \\
\text { the } 2 \text { programs lowered the number } \\
\text { of injuries }\end{array}$ \\
\hline $\begin{array}{l}\text { Donchin et } \\
\text { al, } 1990 \\
(62)^{\mathrm{a}}\end{array}$ & $\begin{array}{l}\text { 3-month intervention } \\
\text { study, } 6 \text { months of } \\
\text { follow-up monthly and } \\
\text { supervision for } 1 \text { year; } \\
\text { secondary prevention }\end{array}$ & $\begin{array}{l}142 \text { nursing staff } \\
\text { with a minimum of } \\
3 \text { episodes of back } \\
\text { problems annually; } \\
\text { questionnaire, } \\
\text { physical capacity } \\
\text { tests }\end{array}$ & $\begin{array}{l}\text { S, PhE, } \\
\text { PSE }\end{array}$ & $\begin{array}{l}\text { equipment } \\
\text { Group A: } 45 \text {-min } \\
\text { exercise sessions for } \\
\text { strengthening back } \\
\text { flexors twice weekly } \\
\text { for } 3 \text { months; group } \\
\text { B: } 5 \times 90 \text {-min back } \\
\text { education sessions; } \\
\text { group C (referents): } \\
\text { no exercise or edu- }\end{array}$ & $\begin{array}{l}\text { Physical capacity, } \\
\text { endurance and } \\
\text { isometric strength, } \\
\text { periods of LBP; } \\
\text { level I }\end{array}$ & $\begin{array}{l}\text { Group A subjects had increased } \\
\text { trunk-flexor strength but not } \\
\text { extensor strength compared with } \\
\text { group B and C subjects; effect } \\
\text { disappeared after } 6 \text { months; the } \\
\text { number of "painful months" in } \\
\text { subsequent year decreased in group } \\
\text { A compared with groups B and C } \\
\text { (4.5/7.3 \& } 7.4 \text { months) }\end{array}$ \\
\hline $\begin{array}{l}\text { Linton et al, } \\
1989(63)^{\mathrm{a}}\end{array}$ & $\begin{array}{l}15 \text { weeks intervention } \\
\text { program plus } 6 \text { months } \\
\text { of follow-up; secondary } \\
\text { prevention }\end{array}$ & $\begin{array}{l}36 \text { licensed practical } \\
\text { nurses or nursing } \\
\text { aides (and } 30 \\
\text { referents) currently } \\
\text { working but with } \\
\text { history of back } \\
\text { problems during the } \\
2 \text { years before } \\
\text { enrollment; } \\
\text { questionnaire, diary, } \\
3 \text { assessments }\end{array}$ & $\begin{array}{l}\text { PhE, } \\
\text { PsE }\end{array}$ & $\begin{array}{l}\text { cation sessions } \\
\text { Group l: behavior } \\
\text { therapy, physical } \\
\text { therapy, "low-back } \\
\text { school", relaxation } \\
\text { training, life-style } \\
\text { factors, strategies } \\
\text { for avoiding injuries } \\
\text { at work; the program } \\
\text { lasted } 8 \text { hours per } \\
\text { day for } 5 \text { weeks; } \\
\text { group C: waiting list } \\
\text { referents; program }\end{array}$ & $\begin{array}{l}\text { Pain intensity, } \\
\text { fatigue, anxiety, } \\
\text { sleep quality, } \\
\text { medication intake, } \\
\text { pain behavior, } \\
\text { activities of daily } \\
\text { living, depression, } \\
\text { helplessness, work } \\
\text { absences for 2-year } \\
\text { period prior to the } \\
\text { study; level I }\end{array}$ & $\begin{array}{l}\text { Intervention subjects showed great- } \\
\text { er improvements than } C \text { subjects in } \\
\text { self-reported problems, anxiety, } \\
\text { sleep quality and fatigue, and activ- } \\
\text { ities of daily living at } 6 \text { months } \\
\text { follow-up; intervention subjects } \\
\text { broke a trend for increasing } \\
\text { amounts of problem-related } \\
\text { absenteeism, while C subjects did } \\
\text { not }\end{array}$ \\
\hline $\begin{array}{l}\text { Cooper et } \\
\text { al, } 1996 \\
(64)\end{array}$ & 6 months & $\begin{array}{l}40 \text { study ward } \\
\text { nurses in high risk } \\
\text { wards and } 118 \\
\text { referents who were } \\
\text { injured but had no } \\
\text { lesion before the } \\
\text { study; } 2 \text { scales (1 } \\
\text { was a visual } \\
\text { analogue scale) }\end{array}$ & $\begin{array}{l}\text { R, PhE, } \\
\text { PSE }\end{array}$ & $\begin{array}{l}\text { not provided } \\
\text { Assessment and } \\
\text { treatment by a } \\
\text { physiotherapist; if } \\
\text { sick leave >4 days, } \\
\text { the injured nurse } \\
\text { was referred for } \\
\text { occupational therapy } \\
\text { assessment, early } \\
\text { return to work with } \\
\text { individually modified } \\
\text { work tasks; reference } \\
\text { ward nurses were } \\
\text { asked to seek care } \\
\text { through their routine } \\
\text { care givers }\end{array}$ & $\begin{array}{l}\text { Subjective problems } \\
\text { in the low back, } \\
\text { disability of daily } \\
\text { activity; level I }\end{array}$ & $\begin{array}{l}\text { A significant decrease in perceived } \\
\text { disability between initial and } \\
\text { tollow-up was found for nurses with } \\
\text { problem scores of }<10 \text { and } \\
\text { reference nurses with problem } \\
\text { scores of }<20 \text {; early intervention } \\
\text { program led to a reduction in } \\
\text { disability so that no relationship } \\
\text { existed between initial disability and } \\
\text { disability at } 6 \text { months after injury }\end{array}$ \\
\hline
\end{tabular}

a Studies included in Bigos et al, 1996 (19).

concerning risk factors for the genesis of work-related low-back problems, as well as preventive strategies for the work force. Many studies were scrutinized by this work group. This scrutiny aimed at finding results which had been received from studies with scientific methods excluding or minimizing the possibilities of bias. In 1996 the scientific committee of the AAOS [in which one of the authors $(\mathrm{TH})$ is a member] published a scientific 
evaluation of these prospective cohort studies of workrelated back problems (19). The studies included in the evaluation are shown in tables 4 and 5 .

\section{Physical factors}

\section{Lifts and transfers and "save the patient" situations}

Many studies have shown a relationship between patientlifting frequency and low-back problems. According to Jensen (20), each stressful patient handling involves some risk of a back injury for the nurse. If the patient behaves as predicted and everything goes smoothly, the risk is minimal. However, if anything unusual happens, for example, the legs of the patient give way or the patient slides out of bed or off a chair (ie, a "save the patient" situation occurs) the risk of low-back problems for the nurse increases dramatically.

In a meta-analysis of 6 epidemiologic studies from 1985-1988 Jensen (20) summarized that the prevalence of low-back problems among the nursing personnel who more frequently handled patients was about 3.7 times that of nursing personnel who infrequently handled patients (table 1). A register study showed an increased risk of operation for herniated lumbar disc among assistant nurses who worked in hospitals or nursing homes where heavy lifting occurred compared with the overall Danish female population $(21)$.

Smedley et al $(11,22)$ reported that the risk of lowback problems was less if lifting devices were used than if not. [See references 11 (table 1) and 22 (table 2).] In another study, however, the use of lifting devices had no positive effect on low-back problems (23), nor was lifting patients in or out of baths with hoists related to lower risks for low-back problems than lifting manually (22). Studies have shown that "positioning a patient in bed" more often than other patient transfer procedures leads to low-back problems among nurses $(24,25)$. Takala \& Kukkonen (26) noted that the work postures were awkward also when lifting devices were used, and, in another study on exposure in nursing work, Vojtecky et al (27) found that whether devices were used or not was related to type of load, stress in the lifting situation, work experience, and the amount of training in the use of devices. As far as the equipment is concerned, beds which are not adjustable in height can be risk factors for low-back problems (28), and for low-back stress (29), as the height of the bed has important consequences on the postures and handling capacity of nurses (30).

\section{Work postures}

Awkward work postures and standing great parts of a day were associated with a double risk of back problems among French nurses, as was also frequent lifting, pulling, and pushing (3). Other studies have also found that lifting and pushing, as well as walking and standing great parts of a day, are of significance for low-back problems $(2,28,30,31,32)$. However, in a study in which nurses from hospitals in Belgium and The Netherlands were studied, it was found that, in spite of the heavier work load among Dutch nurses, the life-time prevalence of low-back problems was higher among Belgian nurses. The work load was defined as time per day spent on strenuous patient-handling tasks (turning, lifting, helping, etc) (33).

\section{Work category and clinical area}

It has been shown in some studies that nurses' aides have a higher prevalence of work-related low-back problems than registered nurses $(3,13,23)$, while a study from the United Kingdom did not reach the same conclusion (11). Low-back problems were more closely related to exposure than to work category according to Stobbe et al (34). Each of the 3 work categories (licensed practical nurses, nurses' aides, and attendants) were dichotomized into those who frequently lifted patients and those who infrequently lifted patients. In all 3 work categories, those who were exposed to frequent lifting had more low-back problems than the other exposure group. Heap (35) showed that the greater amount of sick leave among nurses' aides could be explained by their more strenuous work and that they, to a greater extent than registered nurses, worked in geriatric wards with many patient transfers.

Work in orthopedic, geriatric, and rehabilitation wards with physically demanding nursing tasks has been regarded as a risk factor for low-back problems in several studies $(23,24,33,36)$.

\section{Work organizational factors}

\section{Staff density - work overload - stress}

Staff density has been found to be an important factor in work organization and has been related to low-back problems $(32,36)$. When the staff density is low, the nurses have to work alone frequently, and unassisted lifts were a risk factor for back injuries in a Canadian study (36) Work pressure and a need to slow down at work were related to an increased risk for low-back problems among Dutch nurses. Work disturbed by unseen events was also a risk factor (28).

An insufficient number of staff can lead to work overload, which was seen in several studies to contribute to feelings of stress among nurses $(24,37,38)$. Of nurses with low-back problems, Cato et al (24) showed that $73 \%$ reported feeling overly stressed at work compared with those without low-back problems (53\%). Some of the most important factors which contributed to work stress 
were a feeling of overload, emotional drain, and the perception of conflicting demands and trouble with management $(24,37)$.

\section{Shift work}

One study reported that work on a day shift was a risk factor for low-back problems. This finding may be explained by the higher physical demands, especially in patient handling and nursing care during the days when lifting and bending requirements are higher than during the evenings or nights (9). In another study, shift work versus day work was not a risk factor for low-back problems (11).

Working full-time during a week as compared with part-time was considered a risk factor in some studies (31), but not in others (11).

\section{Mode of nursing care}

Team care as opposed to traditional nursing care means that a nurse has the responsibility to care fully for a few patients instead of shared responsibility for many patients. In a study from 2 geriatric wards the personnel in a modern ward with team care routines performed half as many lifts as the personnel in the traditional ward, irrespective of equivalent patient handling needs. The team-care routines seemed to facilitate the work since the team got to know their "own" patients well. During 4 years $2.5 \%$ in the modern ward and $5 \%$ in the traditional ward reported strain injuries in the back (2). In another cross-sectional study, however, mode of nursing care was not related to low-back problems (39).

\section{Work technique}

No effect of training in patient handling on low-back health was found by Harber et al (40). However, inadequate training was reported by $52 \%$ of the back injured nurses as the cause of the injuries (36). There exists an international controversy concerning "proper" lifting techniques (37), and there is no proof of a particular technique leading to a decrease in low-back problems among nurses. The importance of compliance was seen in an Australian study in which the total weight lifted not in accordance with any of the recommended methods was the main risk factor for back problems. Factors contributing to choice of method were patient cooperation, occupational category, and number of nurses carrying out the lift (41).

\section{Psychosocial factors}

\section{Psychological demands, authority over decisions, skill utilization and social support}

Psychological demands, authority over decisions, skill utilization, and social support at work were related to low-back health in some nursing studies $(13,42,43)$. The relative risk of low-back problems was higher for those with high job strain as compared with those with low job strain. Job strain was defined as a combination of high work demands and limited possibilities to influence work $(42,43)$. In another study, institutional policy (ie, conflicts between own beliefs and those of the institution, lack of power and influence), work overload, and poor social relations were related to low-back problems among full-time nurses in wards with a high perceived ergonomic load. Moreover, responsibility was related to low-back problems for full-time work in wards with low perceived ergonomic load (38). There are, however, studies in which the psychosocial factors were not considered to be risk factors $(39,44)$.

\section{Job satisfaction}

Job satisfaction predicted low-back health in the prospective study by Ready et al. The effect of this factor was evident when combined with earlier severe low-back problems and smoking (45). Attitudes towards health and also psychological disturbance predicted back pain among student nurses (46). In the study of nurses from hospitals in Belgium and The Netherlands that has already been mentioned, Dutch nurses had a heavier job load, but, in spite of this, they experienced higher work satisfaction, had more positive attitudes towards pain, work and activity, and used more positive coping strategies than Belgian nurses. The Dutch nurses report-

Table 4. Summary of the cross-sectional, case-referent, and prospective studies in tables 1 and 2.

\begin{tabular}{|c|c|c|c|c|}
\hline Type of study & Physical factors & $\begin{array}{l}\text { Work organization } \\
\text { factors }\end{array}$ & Psychosocial factors & $\begin{array}{l}\text { Combination of physical and } \\
\text { work organization and psycho- } \\
\text { social factors }\end{array}$ \\
\hline $\begin{array}{l}\text { Cross-sectional studies } \\
(\mathrm{N}=21)\end{array}$ & $\begin{array}{l}2,3,11,13,20,221,24 \\
25,28,30,31,32,33,34 \\
39,41,44\end{array}$ & $\begin{array}{l}2,9,13,24,32,37,38 \\
39,41,44\end{array}$ & $13,33,38,39,43,44$ & $2,13,24,32,33,39,41,44$ \\
\hline Prospective studies $(\mathrm{N}=10)$ & $\begin{array}{l}22,23,{ }^{\mathrm{c}} 35,36,42,44 \\
45,{ }^{\mathrm{b}} 52^{\mathrm{b}}\end{array}$ & $23,36,40,44$ & $40,{ }^{b} 42,44,45,{ }^{b} 46$ & $23,{ }^{b} 36,42,44,45^{b}$ \\
\hline
\end{tabular}

a Included in Jensen 1990 (20) were 5 cross-sectional studies $(34,83,84,85,86)$ and one prospective cohort study (23).

b Included in Bigos et al, 1996 (19). 
ed a lower life-time prevalence of low-back problems than the Belgian nurses did (33).

\section{Summary of work-related factors in the reported studies}

It is evident that, in spite of different study designs, most research points to physical factors as contributing to lowback problems, while the contribution of work organizational and psychosocial factors is not as clear. (For a summary see table 4.)

\section{Individual factors}

In spite of the focus on work-related factors in this review, the individual factors analyzed in some studies are also included. No conclusive relationships have been found between individual factors and low-back problems among nurses. Reviews of the general population or vocational groups have in general shown that low-back problems increase with age up to 50-60 years of age, after which there is a decline $(47,48)$. Hence high age is a risk factor mainly for women (49), a finding which was also shown in some nursing studies $(30,38,39)$ but not in others $(13,23,28,35)$. At the age $40-44$ years the risk of lumbar pain was twice as high as for the group $<35$ years of age among French nurses (44). A literature review has, on the contrary, shown that nurses with lowback problems are often younger than those without these problems (50).

Cigarette smoking as a risk factor for low-back problems also showed a contradictory outcome. Some studies found a relation between smoking and low-back problems among nurses $(45,51)$, while others did not $(9,13)$. In the general population some reviews also showed a contradictory outcome as regards smoking $(47,48)$.

Those who regarded their physical fitness as low, when compared with persons of their own age, had severe low-back problems in a cross-sectional study (13), while this factor did not discriminate between those with or without low-back problems (45) or low-back strength (52).

Out of 199 student nurses who were followed for 20 months, some personal psychological factors were significant in differentiating between cases of back problems and noncases. Moreover, in the more serious cases, physical factors seemed to be more important (46). Among French nurses minor psychiatric disorders were associated with an increased risk of back problems (relative risk 2.7) (3), and among English nurses frequent low mood was associated with low-back problems leading to absence from work (22).

There seems to be a consensus that a previous history of back problems is a risk for subsequent episodes, as shown in both cross-sectional and prospective studies, and in literature reviews $(3,9,22,23,35$, $45,53)$. However, an American prospective cohort study found that prior history of low-back pain or injury did not predict subsequent problems (52).

The issue of work-relatedness in low-back problems often arises. In 2 French studies commuting time to work was studied. In the prospective study, commuting 1 hour or more was a risk factor (44), while in the cross-sectional study commuting more than 1 hour was not a risk factor (3).

\section{Summary of individual factors in the reported studies}

No conclusive relationships have been found between individual factors and low-back problems among nurses. However, a "history of back problems" seems indisputably related to new episodes of low-back problems.

\section{Intervention studies}

Regarding the considerable costs caused by absence from nursing work due to low-back problems among nurses, surprisingly few programs with the aim of reducing such problems have been reported. Most of these studies have focused on the individual person, and in one such study from Sweden nurses and nurses' aides both with and without back problems lowered the absence from work because of low-back problems with light physical exercise during workhours (54) (table 3).

There have been several studies published that have indicated little, if any, decrease in low-back problems as a result of technique training among participants. Two studies have evaluated ergonomic training among student nurses. In the first study the effect of 40 hours of extra training in patient handling skills on subsequent back problems led to no differences in such problems between the training group and the reference group. However, improvement in patient-handling skills was seen in the training group. The subsequent incidence of acute back injuries occurring during work was lower for the skilled nurses, but there was no significant difference between the skilled and unskilled nurses in the incidence of other forms of back problems (55). The other study of student nurses also failed to show an effect of the program on low-back problems after 2 hours of extra ergonomic training each week during 2 years (56)

Another intervention program consisting of patient transfer technique, physical exercise, and stress management was conducted during 3 years, but it did not produce the expected results. At the 1-year follow up, the nurses who used a new technique $(93 \%)$ rated some patient transfers as less physically strenuous than those who did not use the technique. There was, however, no 
decrease in low-back problems among the nurses (57). In another program in which nurses trained in patient transfer technique, the quality of performing patient transfers improved in the study group, but there was no reduction in low-back problems (58).

A few intervention studies have focused on both the organization (work environment) and the individual person. An ergonomic intervention program of nursing assistants in a nursing home showed that training in the use of devices, modifying the toilets and shower rooms, and applying technical aides in patient care were efficient strategies to reduce low-back problems (59). Another program also resulted in a decrease in back injuries and time lost, and it was therefore considered cost-beneficial. The intervention consisted of prompt assessment, treatment, and rehabilitation through modified work for nurses from high-risk wards (60).

Wood (61) reported two programs in the same study, one focused on the individual persons and the other on the organization. The organization intervention consisted of increased contact for the newly injured nurse with the hospital staff, the physician, and persons from the Workers' Compensation Board to prevent the injured nurse from getting lost in the system. Subsequently, there was a decrease in the number of wage-loss claims filed by nurses for back injuries caused by interaction with residents. On the other hand the individual training program was not effective in that sense.

Some studies are secondary prevention programs for nurses with manifest low-back problems. In one such study, intervention consisted of physical exercise sessions and back training sessions. The number of "painful months" in the subsequent year decreased in the training group. It was suggested that this outcome was a result of increased trunk-flexor strength (62). In a Swedish study, nurses at work, but with a history of back problems, took part in a physical exercise program 1 day a week for 4 weeks. Behavior therapy techniques conducted by a psychologist were included in the program to help the subjects achieve better control of their pain. The intervention subjects showed greater improvements than did the reference group in self-reported pain, fatigue, and daily living activities (63). Another study with early intervention for nurses with low-back problems focused on

Table 5. Summary of the intervention studies in relation to level of intervention (individual, organizational or individual and organizational) in table $3(\mathrm{~N}=11)$.

\begin{tabular}{lll}
\hline $\begin{array}{l}\text { Effect on low- } \\
\text { back problems }\end{array}$ & Individual focus & $\begin{array}{l}\text { Organizational and } \\
\text { individual or } \\
\text { organizational focus }\end{array}$ \\
\hline $\begin{array}{ll}\text { Decrease } \\
\text { No decrease }\end{array}$ & $\begin{array}{l}54,62,63^{a}, 5 \\
55,56,57,58,61\end{array}$ & $59,60,61,64$, o \\
\hline
\end{tabular}

a Studies included in Bigos et al, 1996 (19).

${ }^{b}$ Secondary prevention. assessment and therapy by physiotherapists. The nurses with more than 4 weeks of time lost were referred to occupational therapy, which consisted of a physical exercise program until the nurse was suited for modified work. The early intervention program led to a reduction in disability in that no relationship existed between initial disability and disability at 6 months after injury. In the absence of an intervention program, disability at the time of injury predicted disability after 6 months (64). For a summary of the intervention studies see table 5 .

More-detailed information about exposure and outcome has been reported in Swedish (65).

\section{Discussion}

\section{Study design}

Direct comparisons of data from epidemiologic studies are difficult. In tables 1, 2, and 3 quality comments of the studies are noted. Most of the studies have some limitations concerning study design, definition of the sample, assessment exposure or outcome variables or response rates which must be considered when the results are interpreted. Most of the studies are of cross-sectional design, and retrospective data lead to uncertainties, not only about the start of an illness but also about the assessment of earlier work load. Another problem is the recall bias which, in the retrospective studies, might explain several of the risk factors noted in the study. Case subjects may well be more likely to recall previous injuries and arduous work conditions than referents will.

In the studies of nursing personnel that have included reference groups, other groups of the same personnel category are generally used for reference. This procedure may lead to an underestimation of the risk of low-back problems, as most of them have physically strenuous work. It is probable that the risk of low-back problems would be more evident if reference groups did not have similar physically strenuous work.

Another problem which also leads to an underestimation of risk factors for low-back problems is high turnover rates, which are mainly seen among younger nurses (66). Many nurses leave their job because of back problems (66), but other factors also contribute, such as low salary and the lack of sufficient flexibility of workhours (67).

\section{Work-related factors}

A further explanation of reported contradictory results may be that different work-related factors have significance for new episodes and for the maintenance of lowback problems. It is reasonable to assume that low-back problems and their consequences in nursing work, as in any other work, are affected by physical, psychosocial, 
psychological, and social factors, as well as by factors related to insurance claims. In a recent review on risk factors for low-back disorders, Burdorf \& Sorock summarized that job dissatisfaction and low job-decision latitude proved to be important, but the evidence was not consistent across different study designs and studies with different vocational groups (68). In another review (69), Nachemson reported, however, that psychosocial factors were the most significant factors not only for the maintenance of low-back problems, but also for new episodes.

Attitudes towards the experience of pain and also attitudes towards work, worktasks, employers, supervisors, and co-workers can be risk factors for low-back problems and for the consequences and maintenance of these problems (10). In several studies, out of which 2 were prospective cohort studies, attitudes towards pain, work, and satisfaction with work predicted low-back health (33, 45, 46).

Staff density was seen as a risk factor for low-back problems, and an insufficient number of staff was shown to lead to patient transfers being carried out by only 1 nurse when it would be appropriate for 2 nurses to transfer the patient. In the times of downsizing nursing personnel in Sweden, in the beginning of the 1990s, there was an increase from $11 \%$ to $16 \%$ for nurses who transferred patients alone when two were actually needed, and also a tendency towards an increased prevalence of lowback problems was reported (57). Another consequence of an insufficient number of staff is work overload, which, in the long run, can lead to negative stress. Bongers et al (17) suggested that psychosocial factors could directly influence mechanical load through changes in posture and the like, but it also increased stress when the personal capacity to cope with job control and social support was not sufficient. It has been indicated that lack of relaxation may be of significant importance for the development of musculoskeletal disorders. Psychosocial and psychological factors are likely to prevent relaxation when the stress exposure has terminated (70).

An insufficient number of staff must, however, not only be regarded as a risk factor for the nurse, it is highly probable that it is also related to the quality of nursing care and thus is also of importance for the patient.

From this review of nursing work it seems plausible that heavy loads and unfavorable body positions which include "save the patient" situations can be assumed to give rise to injuries to the back among nurses. Work organizational and psychosocial factors and attitudes can thus affect pain perception and injury reporting (71) and, as such, can also be of importance with respect to the return of an injured nurse to work (72). In addition, Wood has shown that the active interest of nurses' supervisors is of great importance to the return of a person to work after an attack of back pain (61). This factor seems to have been neglected in work organization.
Currently, there are 2 difficulties in determining the relative importance of the psychosocial and physical factors related to back injuries among nursing personnel. First, psychosocial factors are usually measured at the individual level, while physical factors are more often measured at the group level, and often with methods with limited precision or accuracy. Second, "objective measures" of aspects of the psychosocial work environment are difficult to develop and are thus rarely used. Objective methods to measure the physical environment are, however, more readily available. Until it is possible to measure most workplace and individual factors with more comparable techniques, it will be difficult to determine their relative importance (73).

\section{Intervention}

An English review of 10 intervention programs to reduce low-back problems among nurses from 1976 to 1992 showed little long-term benefit (16). Pheasant \& Stubbs suggested that nurses were physically overloaded instead of, as widely believed, undertrained (74). When nursing staff do not benefit from training programs, it may be that intervention has lacked the intensity needed to cause the desired change (75) or it may be a matter of inadequacy on the part of the recommended work technique $(16,41,74)$ or a lack of support for the intervention program from top management (61). It is also possible that the individual nurse may already use an acceptable work technique before training. In addition it is seldom evaluated whether or not a newly taught work technique is really used by nurses.

Another issue is that of compliance with the safety rules already known. Supervisors' involvement in the form of feedback has been found to create the highest compliance ratings (61), and the impact of poor supervision of staff safety in respect to handling patients has been acknowledged (76). If the problem is not related to a skill deficiency, the issue becomes supervisory and organizational rather than educational.

One question is whether ergonomic intervention alone is sufficient to decrease low-back problems among nurses. It is probable that psychosocial factors, attitudes, and beliefs, as well as work organizational factors, influence sick leave because of low-back problems, as seen in industry $(77,78)$. Takala \& Kukkonen (26) proposed that back problems in nursing should be ameliorated as follows: nurses should be given training, including recommendations for lifting techniques and body positions, wards must be equipped with lifting devices, and new comprehensive plans should be made for the whole hospital environment, architecture, and work organization. This proposal highlights the importance of the level of intervention, which may focus on the individual nurse or on the organization or on both. As seen in tables 3 and 5 , most intervention programs focus on individual 
nurses. To decrease low-back problems nurses are usually offered training and education meant to lead to behavior modification or a change in attitudes, abilities, or coping strategies. The organizationally focused types of intervention are aimed at improving the nurses' health by means of imposing changes in the work environment. In this review only 4 studies focused on the work environment $(59,60,61,64)$. Wood (61) stressed the importance of combining the 2 types of programs to reach the best possible outcomes. Kilbom (79) also pointed out that, in order to decrease musculoskeletal problems, intervention programs ought to be multidisciplinary because of the complex and multifaceted etiology involved.

It is, however, questionable whether a reduction in low-back problems can really be expected as a direct effect of improved work conditions and work technique. It can also be considered whether ergonomic intervention can lead to a reduction in low-back problems in the shortterm (80). In an English training program the injury rates were initially reduced, but after 18 months the injury rates climbed back to their original level, where they remained, with apparent random fluctuations (74). Perhaps other effects of a program should be studied, for example, satisfaction with changes in the work situation, and, as suggested by Wallace \& Buckle, preventive activities may instead provide opportunities to continue employment despite a low-back problem $(80,81)$.

In future research on low-back problems among nurses, there is a great need to carry out prospective studies with preventive programs that identify and evaluate risk factors; more cross-sectional studies are hardly necessary. However, without increased methodological rigor of these studies, it will not be possible to identify effective intervention methods and confidently encourage their use (82), which would improve the ability to make comparisons both between different study groups and across studies. Most studies in this review have used quantitative methods. A broadening of this perspective and also the use of participative or interview methods to obtain qualitative data might identify factors that contribute to lowback problems among nurses. Wickström (10) also suggested that, to reach an understanding in intervention studies, it is necessary to adopt methods used in research on education.

\section{Concluding remarks}

Nursing work in close contact with patients often involves both heavy loads and unfavorable body positions. It also often includes elements such as "save the patient" situations, for example, those in which the patient's legs give way or the patient is about to fall out of a bed or off a chair. Such situations can cause uncontrollable loads on nursing staff, loads that can irrefutably be assumed to give rise to injuries to the back and other parts of the body. High turnover among young nurses and the use of medical staff as a reference group are factors that may lead to an underestimation of the risk of developing lowback problems.

A considerable number of studies of nursing staff have shown the connection between lifting and transferring patients on one hand and low-back problems on the other. To our knowledge there are no studies that show that work organizational or psychosocial factors, as such, cause low-back problems. However, these factors can be of significance to the consequence and maintenance of low-back problems, by affecting, for example, the perception of pain and the tendency to report illness. Factors in nursing work which might have significance for this connection are staff density and job satisfaction. In this review the single individual factor indisputably related to low-back problems was "history of back problems".

Prevention programs do not show unequivocally positive results. However, in most programs, the level of intervention focuses on the individual person, while lowback problems also undoubtedly need an organizational solution.

\section{References}

1. Kilbom $\AA$. Repetitive work of the upper extremity, part II: the scientific basis of the guide. Int $J$ Ind Ergonomics $1994 ; 14: 59-86$.

2. Ljungberg A-S, Kilbom $\AA$, Hägg G. Occupational lifting by nursing aides and warehouse workers. Ergonomics 1989;32(1):59-78.

3. Estryn-Behar M, Kaminski M, Peigne E, Maillard MF, Peilletier A, Berthier C, et al. Strenuous working conditions and musculoskeletal disorders among female hospital workers. Int Arch Occup Environ Health 1990;62:47- 57 .

4. Calhoun G. Hospitals are high-stress employers. Hospitals $1980 ; 16: 171-6$.

5. Tan CC. Occupational health problems among nurses [review]. Scand J Work Environ Health 1991;17:221 - -30.

6. Arnetz JE, Arnetz BB, Petterson I-L. Violence in the nursing profession: occupational and lifestyle risk factors in Swedish nurses. Work Stress 1996;10(2):119--27.

7. Lees $\mathrm{S}$, Ellis $\mathrm{N}$. The design of a stress-management programme for nursing personnel. J Adv Nurs 1990;(15):946--.. 61.

8. Ekeberg C, Lagerström M, Lützén K. Empowerment and occupational health nursing. AAOHN J 1997;45(7):342-8.

9. Rydén L, Molgaard C, Bobitt S, Conway C, Conway J. Occupational low-back injury in a hospital employee population: an epidemiologic analysis of multiple risk factors of a highrisk occupational group. Spine 1989;14(3):315-20.

10. Wickström G. Natural science and humaniora in intervention research. In: Toomingas A, Bach E, Bast-Pettersen R, Lindström K, Kiviranta J, editors. Research on musculoskeletal disorders among health care personnel. Copenhagen: Tema Nord Nordic Council of Ministers, 1994:15-17.

11. Smedley J, Egger P, Cooper C, Coggon D. Manual handling activities and risk of low back pain in nurses. Occup Environ 
Med 1995;52:160-3 3

12. Biering-Sörensen F. Prospective study of low back pain in the general population: occurrence, recurrence and aetiology. Scand J Rehabil Med 1983;15:71-9.

13. Lagerström M, Wenemark M, Hagberg M, Wigaeus Hjelm E. Occupational and individual factors related to musculoskeletal symptoms in five body regions among Swedish nursing personnel. Int Arch Occup Environ Health 1995;68(1):2735 .

14. Lagerström M, Hagberg M. Evaluation of a 3 year education and training program: for nursing personnel at a Swedish hospital. AAOHN J 1997;45(2):83-92

15. Engkvist I-L, Hagberg M, Lidén A, Malker B. Over-exertion back accidents among nurses' aides in Sweden. Saf Sci 1992;15:97-108.

16. Hignett S. Work-related back pain in nurses. J Adv Nurs 1996;23:1238-46.

17. Bongers PM, de Winter CR, Kompier MAJ, Hildebrandt VH. Psychosocial factors at work and musculoskeletal disease [review]. Scand J Work Environ Health 1993;19:297-312.

18. Kuorinka I, Jonsson B, Kilbom Å, Vinterberg H, BieringSørensen F. Standardized Nordic questionnaire for the analysis of musculoskeletal symptoms. Appl Ergon 1987;18:2337.

19. Bigos S, Holland J, Webster M, Webster J, Bombardier C, Battié MC, et al. Prevention and risks of reporting occupational back problems; a methodologic literature analysis. Presented at the ISSLS Conference, New York, 23 June 1996.

20. Jensen R. Back injuries among nursing personnel related to exposure. Appl Occup Environ Hyg 1990;5:38-45.

21. Jörgensen S, Hein O, Gyntelberg F. Heavy lifting at work and risk of genital prelapse and herniated lumbar disc in assistant nurse. Occup Med 1994;44:46-9.

22. Smedley J, Egger P, Cooper C, Coggon D. Prospective cohort study of predictors of incident low back pain in nurses. BMJ 1997;314:1225-8.

23. Venning P, Walter S, Stitt L. Personal and job related factors as determinants of incidence of back injuries among nursing personnel. J Occup Med 1987;20(10):820-5.

24. Cato C, Olson D, Studer M. Incidence, prevalence, and variables associated with low back pain in staff nurses. AAOHN J 1989;3(8):321-7.

25. Leighton D, Reilly T. Epidemiological aspects of back pain: the incidence and prevalence of back pain in nurses compared to the general population. Occup Med 1995;45(5):263-7

26. Takala E-P, Kukkonen R. The handling of patients on geriatric wards. Appl Ergon 1987;18(1):17-22.

27. Vojtecky M, Harber P, Sayre J, Billet E, Shimozaki S. The use of assistance while lifting. J Safety Res 1987;18:49—56.

28. Engels J, van det Gulden J, Senden T, van't Hof B. Work related risk factors for musculoskeletal complaints in the nursing profession: results of a questionnaire survey. Occup Environ Med 1996;53:636-41.

29. de Looze MP, Zinzen E, Caboor D, Heyblom P, van Bree E, van Roy $P$, et al. Effect of individually chosen bed-height adjustments on the low-back stress of nurses. Scand J Work Environ Health 1994;20:427-34

30. Lee Y-H, Chiou W-K. Risk factors for low back pain, and patient-handling capacity of nursing personnel. J Safety Res 1994;25(3):135-45.

31. Fuortes LJ, Shi Y, Zhang M, Zwerling C, Schootman M. Epidemiology of back injury in university hospital nurses from review of workers compensation records and a casecontrol survey. J Occup Med 1994;36(9):1022-6.
32. Larese F, Fiorito A. Musculoskeletal disorders in hospital nurses: a comparison between two hospitals. Ergonomics 1994;37(7):1205-11.

33. Burton A, Symonds T, Zinzen E, Tillotson KM, Caboor D, Van Roy $P$, et al. Is ergonomic intervention alone sufficient to limit musculokeletal problems in nurses? Occup Med 1997;47(1):25-32.

34. Stobbe T, Plummer R, Jensen R, Attfield M. Incidence of low back injuries among nursing persomnel as a function of patient lifting frequency. J Safety Res 1988;19(1):21—8.

35. Heap D. Low back injuries in nursing staff. J Soc Occup Med 1987;37:66-70.

36. Yassi A, Khokhar J, Tate R, Cooper J, Snow C, Vallentyne S. The epidemiology of back injuries in nurses at a large Canadian tertiary care hospital: implications for prevention. Occup Med 1995;45(4):215-20.

37. Owen BD. Personal characteristics important to back injury. Rehabil Nursing 1986;11(4):12-6.

38. Bru E, Mykletun RJ, Svebak S. Work-related stress and musculoskeletal pain among female hospital staff. Work Stress 1996;10(4):309-21.

39. Skovron ML, Mulvihill MN, Sterling RC, Nordin M, Tougas $\mathrm{G}$, Gallagher $\mathrm{M}$, et al. Work organization and low back pain in nursing personnel. Ergonomics 1987;30(2):359-66.

40. Harber P, Pena L, Hsu P, Billet E, Greer D, Kim K. Personal history, training, and worksite as predictors of back pain of nurses. Am J Ind Med 1994;25:519-26.

41. Lo S, Boldy D, Ha S. A tentative back injury model related to nurses caring for geriatric patients. Socio-Econ Plann: SCi 1993;27(2):131-6.

42. Josephson $M$, Lagerström $M$, Hagberg $M$, Wigaeus Hjelm $E$. Musculoskeletal symptoms and job strain among nursing personnel: a study over a three-year period. Occup Environ Med 1997;54:681-5.

43. Ahlberg-Hultén GK, Theorell T, Sigala F. Social support, job strain and musculoskeletal pain among female health care personnel. Scand J Work Environ Health 1995;21:435-9.

44. Niedhammer I, Lert F, Marne M. Back pain and associated factors in French nurses. Int Arch Occup Environ Health 1994;66:349-57.

45. Ready A, Boreskie S, Law S, Russell R. Fitness and lifestyle parameters fail to predict back injuries in nurses. Can J Appl Physiol 1993;18(1):80—90.

46. Klaber Moffet J, Hughes G, Griffiths P. A longitudinal study of low back pain in student nurses. Int J Nurs Stud 1993;30(3):197-212.

47. Riihimäki $H$. Low-back pain, its origin and risk indicators [review]. Scand J Work Environ Health 1991;17:81-90.

48. Hansson T. Ländryggsbesvär och arbete [Low back problems and work]. Stockholm: Arbetsmiljöfonden, 1989.

49. Nisell R, Vingård E. Arbetsrelaterade sjukdomstillstånd i rörelseorganen: en medicinsk kunskapsöversikt [Work load related disorders of the musculoskeletal system; a review]. Solna (Sweden): National Institute for Working Life, 1992. Arbete och hälsa, no 40. English summary.

50. McAbee R. Nurses and back injuries: a literature review. AAOHN I 1988;36(5):200-9.

51. Owen B, Damron C. Personal characteristics and back injury among hospital nursing personnel. Res Nurs Health 1984; 7:305-13.

52. Mostardi R, Noe D, Kovacik M, Porterfield J. Isokinetic lifting strength and occupational injury: a prospective study. Spine 1992;17(2):189-93.

53. McAbee R, Wilkinson W. Back injuries \& registered nurses. 
AAOHN J 1988;36(3):106 - 12 .

54. Gundewall B, Liljeqvist M, Hansson T. Primary prevention of back symptoms and absence from work. Spine 1993;18(5):587-94.

55. Videman T, Rauhala H, Asp S, Lindström K, Cedercreutz G, Kämppi $M$, et al. Patient-handling skill, back injuries, and back pain: an intervention study in nursing. Spine 1989;14(2):148-56.

56. Hellsing A-L, Linton S, Andershed B, Bergman C, Liew M. Ergonomic education for nursing students. Int J Nurs Stud $1993 ; 30(6) ; 499-510$.

57. Lagerström M, Josephson M, Pingel B, Tjernström G, Hagberg $M$, the Moses Study group. Evaluation of the implementation of an education and training programme for nursing personnel at a hospital in Sweden. Int $\mathbf{J}$ Ind Ergon 1998;21:79-..90.

58. Feldstein A, Valanis B, Stevens W, Overton C. The back injury prevention project pilot study: assessing the effectiveness of back attack, and injury prevention program among nurses, aides, and orderlies. J Occup Med 1993;35(2):114-9.

59. Garg A, Owen B. Reducing back stress to nursing personnel: an ergonomic intervention in a nursing home. Ergonomics 1992;35(11):1353-75.

60. Yassi A, Tate R, Cooper J, Snow C, Vallentyne S, Khokhar J. Early intervention for back-injured nurses at a large Canadian tertiary care hospital: an evaluation of the effectiveness and cost benefits for a two-year pilot project. Occup Med 1995;45(4):209-14.

61. Wood D. Design and evaluation of a back injury prevention program within a geriatric hospital. Spine 1987;12(2):7782.

62. Donchin M, Woolf O, Kaplan L, Floman Y. Secondary prevention of low-back pain: a clinical trail. Spine 1990;15(12):1317-20.

63. Linton S, Bradlev L, Jensen I. The secondary prevention of low back pain: a controlled study with follow-up. Pain 1989;36:197-207.

64. Cooper J, Tate R, Yassi A, Khokhar J. Effect of an early intervention program on the relationship between subjective pain and disability measures in nurses with low back injury. Spine 1996;21(20):2339-6.

65. Lagerström M, Hansson T, Hagberg M. Ländryggsbesvär i sjukvårdsarbete [Low back problems in nursing]. Solna (Sweden): National Institute for Working Life, 1997. Arbete och hälsa, no 22. English summary.

66. Stubbs DA, Buckle PW, Hudson MP, Rivers PM, Baty D. Backing out: nurse wastage associated with back pain. Int $\mathrm{J}$ Nurs Stud 1986;23(4):325-36.

67. Moores B, Singh B, Tun A. An analysis of the factors which impinge on a nurse's decision to enter, stay in, leave or reenter the nursing profession. J Adv Nurs 1983;8:227-35.

68. Burdorf A, Sorock G. Positive and negative evidence of risk factors for back disorders [review]. Scand J Work Environ Health 1997;23:243-56.

69. Nachemson A. Ont i ryggen- orsaker, diagnostik och behan- dling. Stockholm: Statens Beredning för Utvärdering av medicinsk metodik, 1991.

70. Melin B, Lundberg U. A biopsychological approach to workstress and musculoskeletal disorders. J Psychophysiol 1997;11:238-47.

71. McGill S. The biomechanics of low back injury: implications on current practice in industry and the clinic. J Biomechanics $1997 ; 30(5): 465-75$.

72. Linton S. Cognitive-behavioral and psychosocial aspects of musculoskeletal pain problems. In: Toomingas A, Bach E, Bast-Pettersen R, Lindström K, Kiviranta J, ed. Research on musculoskeletal disorders among health care personnel. Copenhagen: Nordic Council of Ministers, 1994:25 7.

73. Bernard B, ed. Musculoskeletal disorders and workplace factors. Cincinnati $(\mathrm{OH})$ : NIOSH, 1997. Publication no 97141.

74. Pheasant S, Stubbs D. Back pain in nurses: epidemiology and risk assessment. Appl Ergon 1992;23(4):226-32.

75. Goldenhar L, Schulte P. Intervention research in occupational health and safety. J Occup Med 1994;37(7):763-75.

76. Love C. Lifting injury: a study of the occupational health perspective. Nurs Stand 1997;11(26):33-8.

77. Burton A, Tillotson K, Main C, Hollis S. Psychosocial predictors of outcome in acute and subacute low back trouble. Spine 1995;20(6):722-8.

78. Symonds T, Burton A, Tillotson K, Main C. Absence resulting from low back trouble can be reduced by psychosocial interventions at the work place. Spine 1995;20(24):2738--45.

79. Kilbom $\AA$. Intervention programmes for work-related neck and upper limb disorders: strategies and evaluation. Ergonomics 1988;31(5):735-47.

80. Kemmlert $\mathrm{K}$. On the identification and prevention of ergonomic risk factors [dissertation]. Solna: National Institute for Working Life, 1997. Arbete och hälsa, 1997:2.

81. Wallace M, Buckle P. Ergonomic aspects of neck and upper limb disorders. Int Rev Ergon 1987;1:173-200.

82. Goldenhar L, Schulte P. Methodological issues for intervention research in occupational health and safety. Am J Ind Med 1996;29:289-94.

83. Mandel J, Lohman W. Low back pain in nurses: the relative importance of medical history, work factors, exercise and demographics. Res Nurs Health 1987;10:165-70.

84. Arad D, Ryan M. The incidence and prevalence in nurses of low back pain. Aust Nurs J 1986;16:44 8 .

85. Sharp G. Occupational induced backpain in nursing staff in a hospital caring for mentally retarded patients. Occupational Health Service Research, Forth Valley Health Board; Royal Infirmary, 1985:201.

86. Gross C, Andree R, Charpnik E. Frequency of lift and back pain among patient handlers. In: 39th annual conference on engineering in medicine and biology. Washington (DC): Alliance for Engineering in Medicine and Biology, 1986:365.

Received for publication: 3 March 1998 